\title{
Efecto del riego por goteo y exudación sobre el rendimiento de hortalizas en clima frío
}

\author{
Effect of drip and exudation irrigation systems on vegetable yield in a cold climate
}

\section{Orlando Edmundo Benavides B. ; Fernando Vicente Barraza A. ${ }^{2}$; Jorge Fernando Navia $E^{3}$.}

\footnotetext{
1 Profesor investigador. I.A. M.Sc. Universidad de Nariño, Pasto. Colombia, orlando.benavides2@gmail.com.

2 Profesor investigador. I.A., Ph.D. Universidad de Córdoba, Montería. Colombia, fbarraza@correo.unicordoba.edu.co.

3 Docente, Ph.D., Universidad de Nariño, Pasto, Colombia, jornavia@gmail.com
}

Citar: BENAVIDES, 0.; BARRAZA, F.; NAVIA, J. 2017. Efecto del riego por goteo y exudación sobre el rendimiento de hortalizas en clima frío. Rev. Cienc. Agr. 34(1): 108-116. doi: http://dx.doi.org/10.22267/rcia.173401.67.

Recibido: Enero 25 de $2016 . \quad$ Aceptado: Mayo 16 de 2016.

\section{RESUMEN}

Los pequeños productores de hortalizas del sur de Colombia, dependen de la precipitación pluvial para el riego de sus cultivos; sin embargo, los periodos de sequía han aumentado, amenazando el rendimiento de las cosechas, por lo tanto, se ha incremento el uso del riego presurizado. El objetivo fue comparar el efecto de los sistemas de riego por goteo y exudación en diferentes ciclos de cultivos sobre el rendimiento de lechuga (Lactuca sativa L.), repollo (Brassica oleracea L. var. Capitata) y brócoli (Brassica oleracea L. var. Italica). Se utilizó un diseño de bloques completos al azar con arreglo bifactorial. El factor 1 comprendió sistemas de riego y un testigo (riego por goteo, cinta exudante y sin riego), mientras que el factor 2 fue representado por dos ciclos de cultivo (primero, entre septiembre 9 y diciembre 12 de 2013 y segundo, entre marzo 11 y junio 13 de 2014). Con sistema de riego por goteo el rendimiento fue mayor para todas las hortalizas. Para lechuga se obtuvieron 20t/ha y presento una disminución de 13\% y 18\% para el tratamiento testigo y riego por exudación, respectivamente. En repollo el rendimiento fue de 53t/ha y presentó disminución del 35\% en los tratamientos testigo y riego por exudación. En brócoli se obtuvieron 13t/ha, donde el tratamiento testigo se redujo en un 59\%, mientras que en el sistema de riego por exudación fue de $40 \%$. Con relación al análisis económico la falta de riego presento relación beneficio costo menor en todos los experimentos.

Palabras clave: Precipitación, minifundista, lechuga, repollo, brócoli. 


\begin{abstract}
Small-scale vegetable crops South of Colombia. However, the increase in dry periods is threatening crop yields, therefore, pressurized irrigation have increased. This study aimed to compare the effect of drip and exudation irrigation systems and different crop cycles on lettuce (Lactuca sativa L.), cabbage (Brassica oleracea L. var. Capitata) and broccoli (Brassica oleracea L. var. Italica). We used the Randomized Complete Blocks design with bi-factorial treatment arrangements. The factor one, corresponds irrigation systems (drip irrigation, exuding hose systems and a control), meanwhile the factor two, comprised 2 planting seasons (the first, between September 9 and December 12 of 2013 and the second March 11 and June 13 of 2014). The drip irrigation system produced the greatest yield in all vegetables. For lettuce, a yield of 20t/ha was obtained, while a reduction of 13 and 18\% was found for the control and exudation irrigation systems, respectively. For cabbage, yield was $53 \mathrm{t} /$ ha and a decrease of $35 \%$ was observed for the control and exudation irrigation systems. For broccoli, 13t/ha were obtained, while in the control and exudation system the yield was reduced by 59\% and 40\% respectively. Finally, the economic analysis showed that the control had the lowest cost/benefit relationship in all experiments.
\end{abstract}

Key words: rainfed, smallholder, lettuce, cabbage, broccoli.

\section{INTRODUCCIÓN}

El departamento de Nariño es el productor de hortalizas de clima frío más importante del suroccidente colombiano. Las principales especies sembradas son papa (Solanum tuberosum L.), tomate (Lycopersicon esculentum Mill.), arveja (Pisum sativum L.), zanahoria (Daucus carota L.), repollo (Brassica oleracea var. Capitata), cebolla junca (Allium fistulosum L.), cebolla cabezona (Allium cepa L.), lechuga (Lactuca sativa L.), coliflor (Brassica oleracea var. Botrytis), ajo (Allium sativum L.), haba (Vicia faba L.) y brócoli (Brassica oleracea var. Italica) (Cárdenas, 2013).

La producción en su mayoría es efectuada por pequeños productores en zonas de minifundio, y dentro del contexto nacional el departamento participa con el $10 \%$ de la producción de lechuga (6215t), 15\% de repollo (10607t) y 12\% de brócoli (1177t), lo que aporta 416738 millones de pesos al producto interno bruto departamental (Romero, 2015). Por esta razón, es importante incorporar tecnologías sostenibles de producción, entre las que se destaca la utilización de sistemas de riego, con el fin de producir en épocas en las que son marcadas las deficiencias hídricas y lograr mayor competitividad.

Uno de los agravantes de la situación actual en cuanto al déficit hídrico, es debido al calentamiento de la temperatura del Océano Pacífico ocasionado por el fenómeno El Niño, donde la zona andina colombiana reporta una reducción de las lluvias entre 20 y $30 \%$ (IDEAM, 2015), lo que ha afectado los cultivos de hortalizas como lechuga, repollo y brócoli, ya que uno de los principales requisitos de producción de estas especies es el adecuado suministro de agua para una buena productividad (Seidel et al., 2017).

Acorde con lo anterior, los sistemas de riego presurizados constituyen una alternativa viable para garantizar la supervivencia y calidad de los cultivos de hortalizas sembrados tanto en invernadero como en campo, debido a que presentan ventajas como ahorro en los costos de mano de obra, mayor cobertura, mejor automatización y 
control de los niveles de presión del agua (Duque, 2015), por lo que es de gran importancia evaluar el efecto de algunos sistemas de riego que se pueden implementar en la zona para conocer sus efectos sobre el rendimiento de hortalizas y determinar cuál brinda más beneficios a nivel agronómico, económico y técnico.

El objetivo de este trabajo fue comparar el efecto que tienen los sistemas de riego por goteo $\mathrm{y}$ exudación sobre el rendimiento de tres especies de hortalizas que se producen en el departamento de Nariño, como lechuga, repollo y brócoli para ofrecer alternativas sostenibles a los pequeños productores de la zona de minifundio.

\section{MATERIALES Y MÉTODOS}

Localización. La investigación se realizó en la Granja Experimental Botana de la Universidad de Nariño, Colombia, ubicada $77^{\circ} 16^{\prime} 33,85^{\prime \prime}$ LO y $01^{\circ} 09^{\prime} 29,86$ "LN, $2820 \mathrm{msnm}$, temperatura promedio mensual $12,4{ }^{\circ} \mathrm{C}$, precipitación promedia anual $694 \mathrm{~mm}$ y humedad relativa promedio $75 \%$ (IDEAM, 2014). De acuerdo con el sistema de clasificación de zonas de vida de Holdridge, corresponde a bosque seco montano bajo (bs-MB).

Conducción del experimento. Se sembraron tres especies de hortalizas: lechuga (cv. Batavia), repollo (cv. Copenhagen Market) y brócoli (cv. De Cicco). Para la siembra de las semillas, se hizo una cama de germinación de 3,0m de largo por 1,0m de ancho, con sustrato preparado con dos partes de arena y una parte de materia orgánica (compost). Cada especie ocupó $1 \mathrm{~m}^{2}$ de la cama de germinación. Las semillas se esparcieron al voleo, a razón de $5 \mathrm{~g} / \mathrm{m}^{2}$ (lechuga), $10 \mathrm{~g} / \mathrm{m}^{2}$ (repollo) y $10 \mathrm{~g} / \mathrm{m}^{2}$ (brócoli). Las plántulas de las tres especies, se trasplantaron a sitio definitivo a los 30 días después de la siembra (dds). Para todas las hortalizas, las distancias de siembra utilizadas fueron $0,4 \mathrm{~m}$ entre surcos y $0,4 \mathrm{~m}$ entre plantas.

La preparación del terreno se realizó 20 días antes del trasplante, mediante una labor de arado y una de rastrillo. Para mejorar las propiedades físicas y químicas del suelo se incorporaron 0.10 t/ha de compost. Se hizo el análisis químico de suelos en el Laboratorio de Suelos Agrícolas de la Universidad de Nariño y de acuerdo con los resultados se hizo fertilización incorporada al momento del trasplante, a $10 \mathrm{~cm}$ de la base de las plantas. Para lechuga, se aplicaron $110 \mathrm{~kg}$ de $\mathrm{N} / \mathrm{ha}$, $30 \mathrm{~kg}$ de $\mathrm{P} / \mathrm{ha}$ y $65 \mathrm{~kg}$ de $\mathrm{K} / \mathrm{ha}$. Tanto para repollo como para brócoli, se aplicaron $180 \mathrm{~kg}$ de $\mathrm{N} / \mathrm{ha}, 40 \mathrm{~kg}$ de $\mathrm{P} / \mathrm{ha}$ y $90 \mathrm{~kg}$ de $\mathrm{K} / \mathrm{ha}$. Las fuentes de fertilizantes utilizadas fueron: urea, superfosfato triple y cloruro de potasio.

Diseño de los sistemas de riego. Se utilizaron dos sistemas de riego: goteo con micro tubos y riego con cinta exudante. Para el abastecimiento de agua en cada sistema, se instaló un tanque elevado de $1000 \mathrm{~L}$ de capacidad, a una altura de $5 \mathrm{~m}$ sobre el nivel del área experimental, con cabeza de presión de 6,3PSI. Como tubería principal en ambos sistemas se utilizó manguera de polietileno de 25,4mm de diámetro y como tubería lateral se utilizó el mismo material, de 19,05mm de diámetro. Los micro tubos para riego por goteo fueron mangueras de polietileno de baja densidad, de $1 \mathrm{~mm}$ de diámetro interno y $1 \mathrm{~m}$ de longitud, con caudal promedio de $3 \mathrm{~L} /$ ha. La separación entre micro tubos fue de 0,4m. Para el caso del sistema de riego por exudación, se utilizó cinta geotextil exudante de $16 \mathrm{~mm}$ de diámetro exterior, con caudal de $3 \mathrm{~h}^{-}$ ${ }^{1} \mathrm{~m}^{-1}$ colocada en la superficie del suelo, a $0,10 \mathrm{~m}$ de distancia de la base de las plantas. Se determinó la infiltración del suelo como variable fundamental para la aplicación del riego mediante el método de doble anillo infiltrómetro (Weber, 2014). En la determinación de la lámina neta de reposición para 
riego de los cultivos se consideraron constantes el umbral de riego (20\%), humedad volumétrica en capacidad de campo $(30,8 \%)$ y punto de marchitez permanente (14\%). El riego se aplicó cuando los tensiómetros instalados a $0,15 \mathrm{~m}$ por debajo de la superficie del suelo, alcanzaban valores de tensión matricial entre 10 y $15 \mathrm{kPa}$.

Diseño experimental. Se realizaron tres experimentos uno con cada especie hortícola, para su análisis se utilizó un diseño de Bloques Completos al Azar con arreglo bifactorial $2 \times 3$. El factor uno corresponde a sistemas de riego (riego por goteo, cinta exudante y sin riego), mientras que el factor dos fue representado por dos ciclos de cultivo (el primero, entre septiembre 9 y diciembre 12 de 2013 y el segundo, entre marzo 11 y junio 13 de 2014). Para un total de seis tratamientos con tres repeticiones. La superficie total del experimento fue de $660,4 \mathrm{~m}^{2}$, donde se establecieron 54 parcelas que ocuparon una superficie de $244,6 \mathrm{~m}^{2}$.

\section{Variables estudiadas y análisis estadístico}

Rendimiento. A los 96 días después del trasplante se tomó el peso unitario (expresado en $\mathrm{kg}$ ) y peso total de la parcela, con estos datos se calculó el rendimiento en $\mathrm{t} / \mathrm{ha}$ de cada hortaliza (lechuga, repollo y brócoli). A los datos obtenidos para peso unitario y rendimiento se les realizó Análisis de Varianza y pruebas de comparación de medias de Tukey $(\alpha \leq 0,05)$ mediante el software SAS 9.0. (SAS Institute Inc. 2002).

Análisis económico. A partir de la información de ingresos totales y costos totales obtenida durante la investigación, se calculó la relación beneficio/ costo $(B / C)$ de los sistemas de irrigación utilizados, mediante la siguiente ecuación (López, 2007):

$$
\frac{B}{C}=\frac{\text { Ingresos totales }}{\text { Costos totales }}
$$

\section{RESULTADOS Y DISCUSIÓN}

Lechuga. En la Tabla 1 se observa que el mayor rendimiento promedio se obtuvo en las dos épocas de siembra con el sistema de riego por goteo, y se encontraron diferencias estadísticas significativas con respecto a los tratamientos testigo y riego por exudación, los cuales disminuyeron su rendimiento en relación al sistema de riego por goteo en 13 y $17 \%$, respectivamente.

De acuerdo con lo anterior, la mayor producción obtenida puede estar relacionada con la aplicación constante de agua que se logra con el sistema de riego por goteo, ya que el cultivo de lechuga posee raíces de poca profundidad que le confiere sensibilidad al déficit hídrico, y en la medida que la aplicación de agua fue localizada cerca de la zona radicular, presentó más eficiencia en la actividad fisiológica de la planta, lo que influye en el crecimiento y desarrollo, en concordancia Kizil et al. (2012).

Tabla 1. Rendimiento ( $\mathrm{t} / \mathrm{ha}$ ) de tres especies de hortalizas cultivadas con sistema de riego por goteo y sistema de riego por exudación, en dos épocas de siembra.

\begin{tabular}{|c|c|c|c|c|}
\hline \multirow{2}{*}{ Especie } & \multirow{2}{*}{$\begin{array}{c}\text { Época de } \\
\text { siembra }\end{array}$} & \multicolumn{3}{|c|}{ Rendimiento } \\
\hline & & T0 & T1 & $\mathbf{T} 2$ \\
\hline \multirow{3}{*}{ Lechuga } & 1 & $17,29 \mathrm{~b}$ & $19,72 \mathrm{a}$ & $16,56 \mathrm{~b}$ \\
\hline & 2 & $18,24 \mathrm{~b}$ & $20,82 \mathrm{a}$ & $16,89 \mathrm{~b}$ \\
\hline & Promedio & $17,77 \mathrm{~b}$ & $20,27 \mathrm{a}$ & $16,73 \mathrm{~b}$ \\
\hline \multirow{3}{*}{ Repollo } & 1 & $34,50 \mathrm{~b}$ & $50,16 \mathrm{a}$ & $55,05 \mathrm{a}$ \\
\hline & 2 & $36,19 \mathrm{~b}$ & $55,98 \mathrm{a}$ & $54,50 \mathrm{a}$ \\
\hline & Promedio & $35,35 \mathrm{~b}$ & $53,07 \mathrm{a}$ & $54,78 \mathrm{a}$ \\
\hline \multirow{3}{*}{ Brócoli } & 1 & $4,96 \mathrm{c}$ & $16,84 \mathrm{a}$ & $7,26 \mathrm{~b}$ \\
\hline & 2 & $5,57 \mathrm{c}$ & $9,06 \mathrm{a}$ & $7,90 \mathrm{~b}$ \\
\hline & Promedio & $5,27 \mathrm{c}$ & $12,95 \mathrm{a}$ & $7,58 \mathrm{~b}$ \\
\hline
\end{tabular}

T0: cultivos cuya única fuente de agua de riego fue la precipitación pluvial, T1: cultivos con riego por goteo con micro tubos y T2: cultivos con riego por cinta exudante. Medias con la misma letra son iguales. 
Respecto al peso unitario de lechuga, en la Tabla 2 se observa que con el tratamiento de riego por goteo se obtuvo $16 \%$ más de peso y superó con diferencias estadísticas significativas a los tratamientos de exudación y testigo. Esto muestra que el menor suministro de agua que hubo con estos dos tratamientos, se manifestó en la reducción del crecimiento de las hojas, observación que concuerda por Abubakari et al. (2011).

En la Figura 1 se observa que el rendimiento del cultivo de lechuga no estuvo afectado por la época de siembra, lo cual indica que en la zona se puede sembrar esta especie durante todo el año mientras se efectúe suministro de riego, principalmente en épocas de escasa precipitación pluvial, evitando la considerable reducción del rendimiento y acumulación de materia seca total que puede ocurrir cuando se presentan condiciones de sequía (Abubakari et al., 2011).
Tabla 2. Peso unitario (kg) de lechuga, repollo y brócoli cultivados con sistemas de riego por goteo y sistema de riego por exudación en dos épocas de siembra.

\begin{tabular}{|c|c|c|c|c|}
\hline \multirow{2}{*}{ Especie } & \multirow{2}{*}{$\begin{array}{l}\text { Época de } \\
\text { siembra }\end{array}$} & \multicolumn{3}{|c|}{ Peso unitario } \\
\hline & & T0 & $\mathrm{T} 1$ & $\mathrm{~T} 2$ \\
\hline \multirow{3}{*}{ Lechuga } & 1 & $0,48 \mathrm{~b}$ & $0,55 \mathrm{a}$ & $0,46 \mathrm{~b}$ \\
\hline & 2 & $0,48 \mathrm{~b}$ & $0,58 \mathrm{a}$ & $0,49 \mathrm{~b}$ \\
\hline & Promedio & $0,48 \mathrm{~b}$ & $0,57 \mathrm{a}$ & $0,48 \mathrm{~b}$ \\
\hline \multirow{3}{*}{ Repollo } & 1 & $0,96 \mathrm{~b}$ & $1,39 \mathrm{a}$ & $1,53 \mathrm{a}$ \\
\hline & 2 & $1,01 \mathrm{~b}$ & $1,55 \mathrm{a}$ & $1,52 \mathrm{a}$ \\
\hline & Promedio & $0,99 \mathrm{~b}$ & $1,47 \mathrm{a}$ & $1,53 \mathrm{a}$ \\
\hline \multirow{3}{*}{ Brócoli } & 1 & $0,14 \mathrm{c}$ & $0,47 \mathrm{a}$ & $0,20 \mathrm{~b}$ \\
\hline & 2 & $0,15 b$ & $0,25 \mathrm{a}$ & $0,22 \mathrm{~b}$ \\
\hline & Promedio & $0,15 \mathrm{c}$ & $0,36 \mathrm{a}$ & $0,21 \mathrm{~b}$ \\
\hline
\end{tabular}

T0: cultivos cuya única fuente de agua de riego fue la precipitación pluvial, T1: cultivos con riego por goteo con microtubos y T2: cultivos con riego por cinta exudante. Medias con la misma letra son iguales.

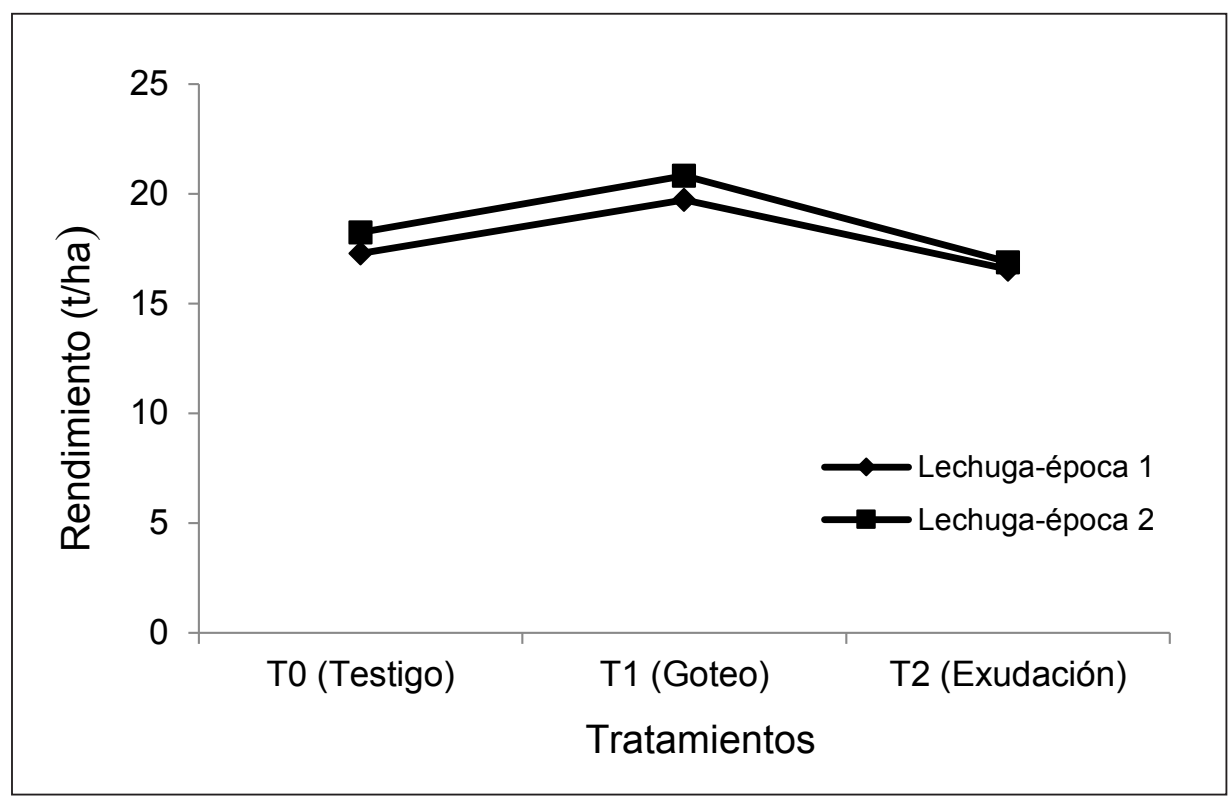

Figura 1. Interacción entre época de siembra de lechuga (Lactuca sativa L.) y sistemas de riego por goteo y exudación. 


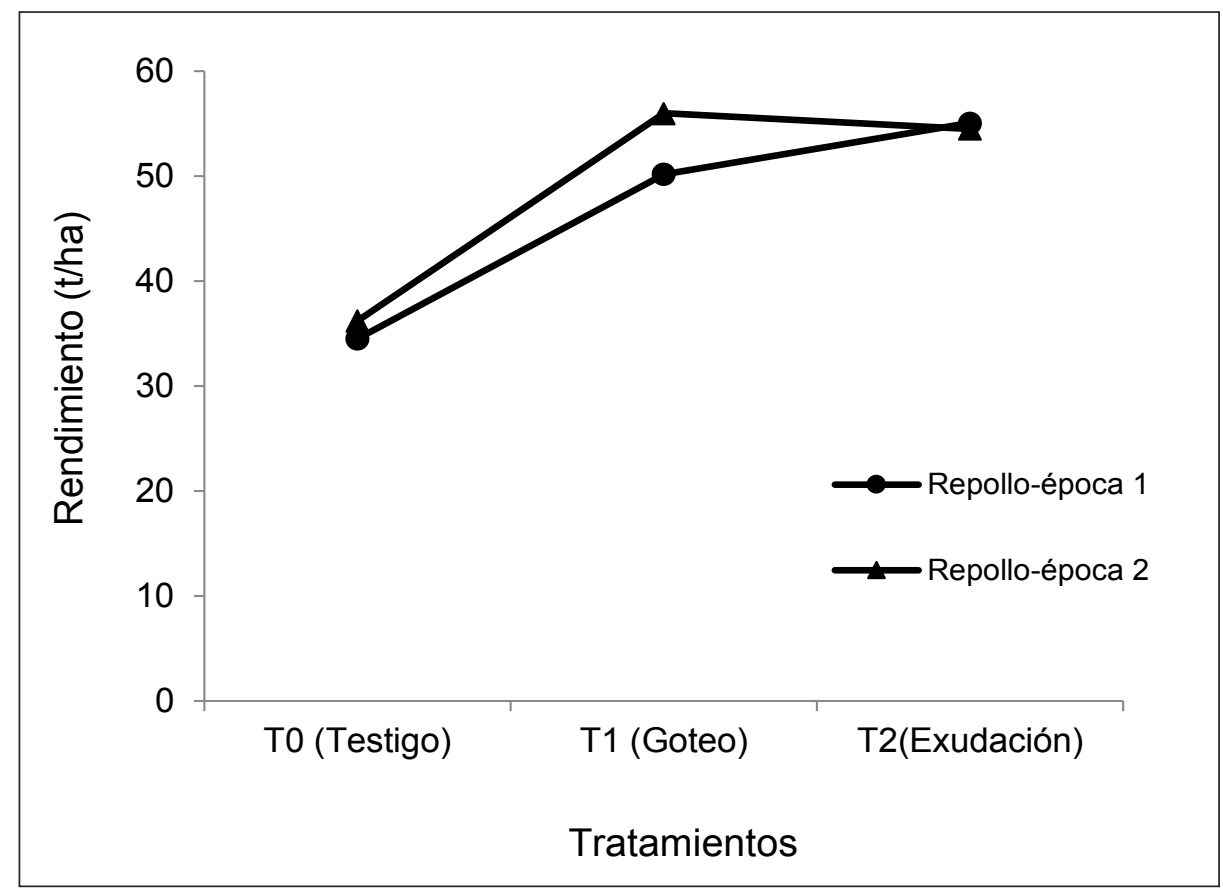

Figura 2. Interacción entre la época de siembra de repollo (Brassica oleracea L. var Capitata) y sistemas de riego por goteo y exudación.

Repollo. De acuerdo con la Tabla 1, se encontró que el tratamiento testigo presentó un 35\% menos de rendimiento con respecto a los tratamientos de riego por goteo y exudación, con los cuales tuvo diferencias estadísticas significativas.

Según la Figura 2, se observa que durante la época de menor precipitación (segunda) el cultivo presentó 11\% más de rendimiento cuando se utilizó el sistema de riego por goteo; por lo tanto, este sistema puede utilizarse para obtener mayor rendimiento, en comparación con métodos tradicionales como el de surcos por gravedad (Xu y Leskovar, 2014), dadas las ventajas como uniformidad en la aplicación y alto uso eficiente del agua (Pragna et al., 2016).

El rendimiento de repollo está influenciado por los métodos de irrigación utilizados y según Ignatius et al. (2013), se han encontrado en ciertas diferencias en la eficiencia, desde valores bajos (33\% para los métodos de gravedad, donde se pierde $67 \%$ del agua aplicada) hasta valores altos (90\% para goteo, donde se utiliza más de $70 \%$ del agua).

Respecto al peso unitario, los tratamientos con sistema de riego por goteo y exudación superaron en un 34\% al testigo, con el que tuvieron diferencias significativas. La menor aplicación de agua que se hizo con el tratamiento testigo condujo a mala calidad, en las cuales según Xu y Leskovar (2014) se afecta el porcentaje de humedad, peso y diámetro.

Brócoli. Como se observa en la Tabla 1, se obtuvo el mayor rendimiento promedio con el sistema de riego por goteo, con diferencias estadísticas significativas respecto a los sistemas por exudación y testigo, a los que superó en 60 y 41\%, en su orden.

Con referencia al peso unitario, en la Tabla 2 se observa que con riego por goteo se presentaron mayores valores promedio, con diferencias estadísticas significativas en comparación con los sistemas de exudación y testigo, que fueron 42 y $58 \%$ respectivamente, menores en su magnitud, en comparación con el sistema de goteo. 
Los métodos de riego utilizados influyeron en el rendimiento y sus componentes (Kumar y Senseba, 2008), dado que los mayores valores de rendimiento y peso unitario que se obtuvieron con riego por goteo muestran el efecto positivo de este sistema, y según Tangune et al. (2016) existe un uso más eficiente de agua, se evita la reducción del rendimiento por excesiva aplicación de agua, o limitación en el crecimiento y acumulación de materia seca por deficiente suministro (Hussain et al., 2016).

La Figura 3 muestra que con el sistema de riego por goteo hubo menor rendimiento en la segunda época de siembra $(9,06 \mathrm{~kg} / \mathrm{ha})$ respecto a la primera $(16,84 \mathrm{~kg} / \mathrm{ha})$. Esto coincide con un aumento de 206mm de precipitación pluvial en el 2014, en comparación con 2013 (Arango et al., 2015), que propició periodos de excesiva humedad, donde se afecta, negativamente la producción (Erdem et al., 2010).

Análisis económico. En la Tabla 3 se observa que los costos correspondientes a los sistemas de riego por goteo y exudación fueron superiores a los del tratamiento testigo, lo que implica que la instalación de sistemas de aplicación de agua para riego de los cultivos de hortalizas requiere una inversión importante y además constituye un componente fundamental del sistema de producción cuando las precipitaciones son irregulares y la fuente de captación de agua es escasa ( Himanshu et al., 2013).

De acuerdo con los resultados obtenidos, se pudo establecer que el tratamiento de riego por goteo presentó mayor relación B/C para los cultivos de lechuga y brócoli, mientras que con el sistema de exudación en cultivo de repollo se obtuvo la mayor relación B/C (Tabla 3). Así, para los cultivos de lechuga y brócoli, el riego por goteo puede ser una entrada de gestión fundamental para garantizar el estado óptimo de humedad en el suelo, debido a que proporciona a cada planta la cantidad de agua que necesita para su normal crecimiento y desarrollo, lo que conlleva al óptimo rendimiento, eficiencia en el uso del agua y beneficios económicos según lo indican De Oliveira et al. (2016).

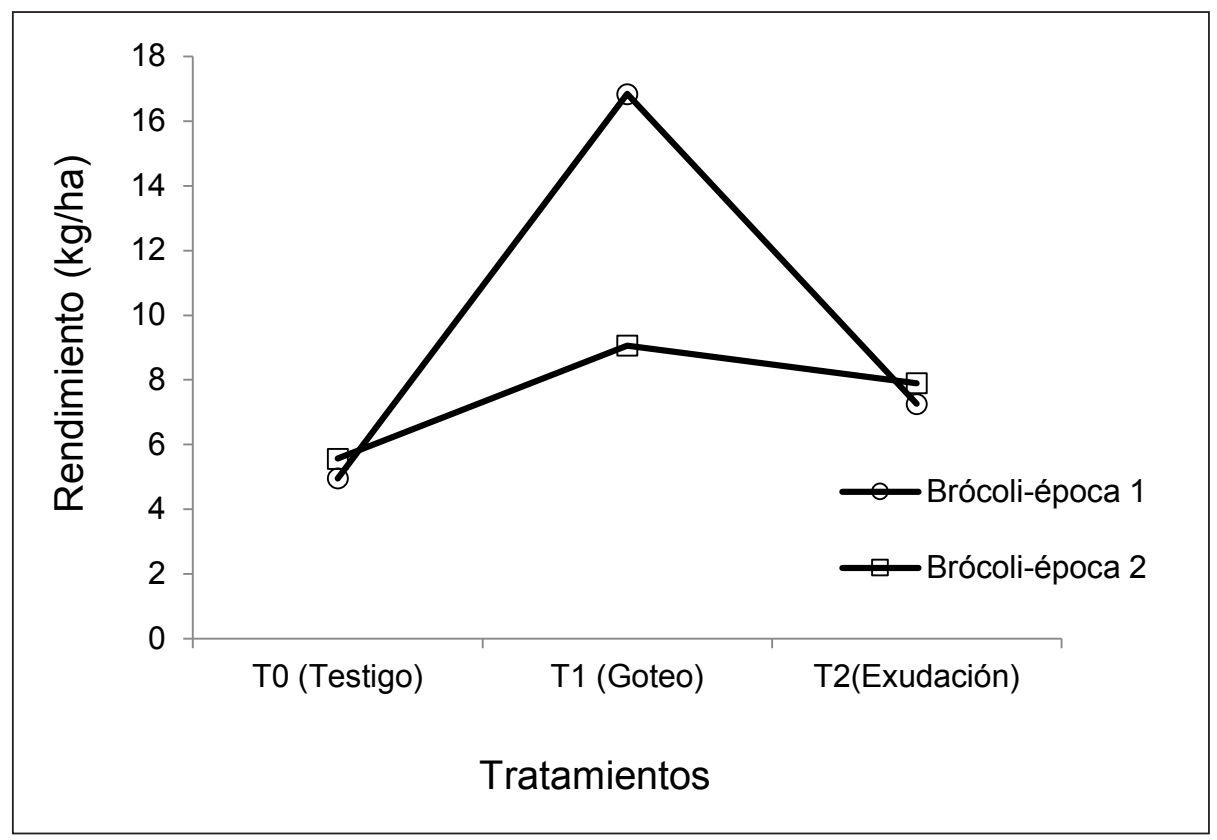

Figura 3. Interacción entre la época de siembra de brócoli (Brassica oleracea L. var Italica) y sistemas de riego por goteo y exudación. 
Tabla 3. Relación beneficio/costo (B/C) para cultivos de lechuga, repollo y brócoli con sistemas de riego por goteo y sistema de riego por exudación.

\begin{tabular}{clrrr}
\hline \multirow{2}{*}{ Cultivo } & \multicolumn{1}{c}{ Variable } & \multicolumn{3}{c}{ Valor anual por sistema de riego (\$/ha) } \\
\cline { 3 - 5 } & Costos & \multicolumn{1}{c}{ T0 } & \multicolumn{1}{c}{ T1 } & \multicolumn{1}{c}{ T2 } \\
\hline \multirow{3}{*}{ Lechuga } & $3,629,000$ & $4,424,726$ & $5,187,000$ \\
& Ingreso bruto anual & $14,216,000$ & $24,324,000$ & $20,076,000$ \\
& Ingreso neto anual & $10,635,000$ & $19,899,274$ & $16,067,000$ \\
& Relación B/C & 2,9 & 4,5 & 3,1 \\
\hline \multirow{3}{*}{ Repollo } & Costos & $3,789,000$ & $4,656,726$ & $5,419,869$ \\
& Ingreso bruto anual & $14,140,000$ & $31,836,000$ & $33,030,000$ \\
& Ingreso neto anual & $10,351,000$ & $27,179,274$ & $27,610,341$ \\
& Relación B/C & 2,7 & 5,8 & 5,9 \\
\hline \multirow{3}{*}{ Brócoli } & Costos & $3,629,000$ & $4,424,726$ & $5,187,000$ \\
& Ingreso bruto anual & $4,208,000$ & $19,425,000$ & $11,370,000$ \\
& Ingreso neto anual & $2,579,000$ & $17,000,274$ & $8,182,331$ \\
& Relación B/C & 0,7 & 3,8 & 1,6 \\
\hline
\end{tabular}

T0: cultivos cuya única fuente de agua de riego fue la precipitación pluvial, T1: cultivos con riego por goteo con micro tubos y T2: cultivos con riego por cinta exudante.

\section{CONCLUSIONES}

En los cultivos de lechuga, repollo y brócoli los mejores rendimientos se obtuvieron con riego por goteo.

Con relación al análisis económico, la falta de riego presento una relación beneficio costo menor en todos los experimentos.

\section{REFERENCIAS BIBLIOGRÁFICAS}

ABUBAKARI, A.; NYARC0, G.; S. MAALINYUUR. 2011. Preliminary studies on growth and fresh weigth of lettuce (Lactuca sativa) as affected by clay pot irrigation and spacing. Pak. J. Biol. Sci. 14(14):747 - 751. doi: 10.3923/pjbs.2011.747.751.

ARANGO, C.; DORADO, J.; GUZMÁN, D.; RUIZ, J. 2015. Climatología trimestral de Colombia. En: IDEAM, http:// www.ideam.gov.co/documents/21021/21789/Clim atolog\%C3\%ADa+Trimestral+para+Colombia+(Ruiz ,+Guzman,+Arango+y+Dorado).pdf/c2825963-c373- 449a-a7cb-8480874478d9. 19 p; consulta: diciembre, 2016.

CÁRDENAS, G. 2013. Acuerdo de competitividad de la cadena de hortalizas. En: Investigación aplicada al desarrollo rural. En: http://conectarural.org/sitio/ material/acuerdo-de-competitividad-de-la-cadenade-hortalizas. 53 p; consulta: octubre, 2015.

DE OLIVEIRA; GEISENHOFF, L.; ALMEIDA, A.; DE LIMA, J.; LAVANHOLI, R. 2016.

Economic feasibility of irrigation systems in broccoli crop. Eng. Agríc. Jaboticabal. 36(3):460 - 468. doi: http://dx.doi.org/10.1590/1809-4430-Eng.Agric. v36n3p460-468/2016.

DUQUE, I. 2015. Sistemas de riego, beneficio clave para el agro colombiano. En: Contexto ganadero, http:// contextoganadero.com/reportaje/sistemas-de-riegobeneficio-clave-para-el-agro-colombiano. 1 p; consulta: octubre, 2015. 
ERDEM, Y.; ARIN, L.; ERDEM, T.; GÜLTAS, H. 2010. Crop water stress index for assessing irrigation scheduling of drip irrigated broccoli (Brassica oleracea L. var. italica). Agricultural Water Management. 98(1):148 - 156. doi: http://10.1016/j.agwat.2010.08.013.

HIMANSHU, S.; SINGH, A.; KUMAR, S.; KALURA, P. 2013. Response of broccoli to irrigation scheduling and methods under drip, sprinkler and surface irrigation. International Journal of Engineering and Advanced Technology. 2(4):777 - 782.

HUSSAIN, M.; RANNU, R.; RAZZAK, M.; AHMED, R.; SHEIKH, M. 2016. Response of broccoli (Brassica oleracea L.) to different irrigation regimes. The Agriculturists. 14(1):98 - 106.

IGNATIUS, M.; IMTIYAZ, M.; KUMAR, J. 2013. Response of cabbage (Brassica Oleracea) under variable irrigation and lateral spacing. Res. J. Engineering Sci. 2(10):1 - 9.

IDEAM. INSTITUTO DE HIDROLOGIA, METEOROLOGIA YESTUDIOSAMBIENTALES. 2014.Cartas climatológicas de Pasto, Nariño. En: Climatografía de las principales ciudades. http://bart.ideam.gov.co/cliciu/pasto/pasto. htm.; consulta: diciembre, 2014.

IDEAM. INSTITUTO DE HIDROLOGIA, METEOROLOGIA Y ESTUDIOS AMBIENTALES. 2015. Por fenómeno de El Niño, ha llovido menos en regiones Andina y Caribe. En: Cambio climático. http://www.minambiente. gov.co/index.php/normativa/126-noticias-cambioclimatico/1770-por-fenomeno-de-el-nino-ha-llovidomenos-en-regiones-andina-y-caribe; consulta: octubre, 2016.

KIZIL, Ü.; GENÇ, L.; INALPULAT, M.; ŞAPOLYO, D.; MIRIK, M. 2012. Lettuce (Lactuca sativa L.) yield prediction under water stress using artificial neural network (ANN) model and vegetation indices. Žemdirbystė=Agriculture. 99(4):409 - 418.

KUMAR, J.; SENSEBA, T. 2008. Yield, irrigation production efficiency and economic return of broccoli (Brassica oleracea Var. Italica) under different irrigation methods and schedules. J. Env. Res. Dev. 2(4):513 - 522.
LÓPEZ, R. 2007. Análisis Costo-Beneficio de la implementación de obras de conservación de suelos sobre parcelas productivas dentro las cuencas Pajcha y Pintu Mayu. Primera edición. Programa Manejo Integral de Cuencas. Cochabamba. 27p.

PRAGNA, G.; KUMAR, M.; SHANKAR, S. 2016. Effect of dripper discharge rates and irrigation schedules on yield of cabbage (Brassica oleracea L var. capitata). Int. J. of Life Sciences. 4(4):554 - 562.

ROMERO, C. 2015. Perfil económico: Departamento de Nariño. En: Perfiles económicos por departamentos, http://www.mincit.gov.co/publicaciones. php?id=16724; consulta: octubre, 2015.

SAS INSTITUTE INC. 2002. Statistical Analysis System. The SAS $^{\odot}$ system 9 for Windows ${ }^{\odot}$. The Power to Know. Cary, NC, 27513, USA. En: https://support.sas.com/ documentation/onlinedoc/91pdf/sasdoc_91/stat_ ug_7313.pdf; consulta: diciembre, 2014.

SEIDEL, S.; WERISCH, S.; SCHÜTZE, N.; LABER, H. 2017. Impact of irrigation on plant growth and development of white cabbage. Agricultural Water Management. 187:99 - 111. doi: http://doi.org/10.1016/j. agwat.2017.03.011.

TANGUNE, B.; MAGELA, G.; DE SOUSA, R.; FREES, R. 2016. Response of broccoli to soil water tension under drip irrigation. Ciências Agrárias. 37(1):7 - 16. doi: http://10.5433/1679-0359.2016v37n1p7.

WEBER, J. 2014. Parámetros del modelo de infiltración de Horton obtenidos mediante el uso de un simulador de lluvia, Córdoba, Argentina. Rev. Ambient. Água. 9(1):161 - 172. doi: http://10.4136/1980-993X.

XU, C.; LESKOVAR, D. 2014. Growth, physiology and yield responses of cabbage to deficit irrigation. Hort. Sci. (Prague). 41(3):138 - 146. 